\title{
Investigation of the effect of brexpiprazole on acute or relapse schizophrenia using the positive and negative syndrome scale: an experimental report
}

\author{
Ishida Tetsuro 1, 2 *, Murayama Tomonori 3, 4
}

${ }^{1}$ Department of Psychiatry, Hokujinkai ISHIBASHI Hospital, Nagahashi, Otaru, Japan.

${ }^{2}$ Department of Neuropsychiatry School of Medicine, Sapporo Medical University, Chuo-ku, Sapporo, Japan.

${ }^{3}$ Graduate school of Medicine, Sapporo Medical University, Chuo-ku, Sapporo, Japan.

${ }^{4}$ Department of Psychiatry, Kushiro Red Cross Hospital, Shinei-cho, Kushiro, Japan.

${ }^{*}$ Corresponding author: Ishida Tetsuro. Ishibashi Hospital 3-7-7-Nagahashi. Zip Code: 047-0036-Japan. Phone: +81-134-25-6655. E-mail: teturoisida@yahoo.co.jp.

Research Ethics Committee Approval (if necessary): This study was approved by the Ethics Committee of Hokujinkai Ishibashi hospital (\#2019001).

Received on: Jan 25, 2022. Accepted on: Feb 8, 2022. Available online: Feb 12, 2022.

\section{Abstract}

Brexpiprazole (BPZ) is a novel antipsychotic drug in the category of serotonin-dopamine activity modulators (SDAMs). It is expected to be effective in the treatment of schizophrenia because of its low side effects and its ability to reduce psychosis. However, clinical data in Japan are still insufficient. We prescribed BPZ to 41 schizophrenic patients; 9 of the 41 dropped out and 32 were retrospectively reviewed for psychiatric symptoms using the positive and negative syndrome scale (PANSS). The results showed that there was significant improvement in the total score, the positive scale, the negative scale and the general psychopathology scale, all after 4 weeks. The improvement started after 1 week for the total score, the positive scale and the general psychopathology scale, and after 2 weeks for the negative scale. The results suggest that $\mathrm{BPZ}$ is effective not only for positive symptoms in the acute phase, but also for negative symptoms after the subacute phase. Further studies are needed to investigate the efficacy of BPZ by patient's characteristics as well as by sub-items of the PANSS.

Keywords: Brexpiprazole; Positive and negative syndrome scale (PANSS); Schizophrenia; Antipsychotics.

\section{Introduction}

Brexpiprazole (BPZ) is the second D2 receptor partial agonist antipsychotic drug in the world after aripiprazole (APZ). BPZ acts as a partial agonist at 5HT1A and D2 receptors and as an antagonist at 5-HT2A receptors, 
modulating serotonin-dopamine neurotransmission. Therefore, BPZ is classed in a new category of serotonindopamine activity modulator (SDAM). BPZ was approved in the US in 2015 for two indications: adjunctive treatment of major depression in adults and schizophrenia. It was approved in Canada in 2017 and in Japan in 2018 for the indication of schizophrenia in adults [1].

The neurochemical profile shows that $\mathrm{BPZ}$ is equivalent to $\mathrm{APZ}$ in its efficacy. It has also been shown that BPZ may have fewer side effects, such as akathisia and extrapyramidal side effects (EPS), than APZ [2]. However, as it is still a young drug in Japan, there is insufficient data and discussion on its actual clinical use.

In this article, we reportour findings from prescribing $\mathrm{BPZ}$ to patients with acute or relapse schizophrenia who are assessed as requiring a change or addition of antipsychotics.

\section{Methodology}

We prescribed BPZ to 41 schizophrenic patients, starting with 1 mg. For some patients, BPZ was their first antipsychotic, while for others it was in combination with or substituted for other antipsychotics. If the need and tolerability of BPZ were confirmed by the attending physician more than one week after initiation, the BPZ dose was increased to $2 \mathrm{mg}$. We evaluated the
Positive and Negative Syndrome Scale (PANSS) at baseline and after 1, 2, 3 and 4 weeks in 32 patients, excluding 9 patients who dropped out midway through the study.

The PANSS was scored by the prescribing physician based on interviews with the patients and their families and caregivers. Wilcoxon's signed-rank test was used for statistical analysis. P-value $<0.05$ was considered significant.

\section{Results (Review)}

Their top five ranking of antipsychotic medications taken prior to BPZ start was: 1st: risperidone $(85.4 \%=35 / 41$ patients), 2 nd: quetiapine $(68.3 \%=28 / 41$ patients), 3rd: olanzapine $(39.0 \%=16 / 41$ patients), 4 th: aripiprazole $(17 \%=7 / 41 \quad$ patients): Aripiprazole $(17 \%=7 / 41)$, 5th: no previous antipsychotic medication before starting BPZ $\quad(14.6 \%=6 / 41)$. Note that some patients were taking more than one antipsychotic medication, so the total value exceeded $100 \%$.

Of the total of 41 patients evaluated in this study, nine dropped out, six because of akathisia and three because of lightheadedness. The prescription dose of BPZ was $1 \mathrm{mg}$ for the whole time (including the nine who dropped out) in 20 patients and increased to $2 \mathrm{mg}$ in 21 patients. The gender of the patients was 16 males and 25 females. Inpatients/Outpatients were 14 inpatients and 27 outpatients. 
The ages of the patients were 10 19 years old (2), 20 29 years old (6), 30 39 years old (4), 40 49 years old (10), 50 59 years old (10), 60 69 years old (5), 70 79 years old (3), 80 89 years old (1) and 90 99 years old (1).

After four weeks of BPZ treatment, the PANSS showed mean total score $(117.38 \pm 11.74$ vs. $93.01 \pm$ 10.97; $p<0.01$ ) (Figure 1), mean positive scale $(30.09 \pm 4.18$ vs. $20.10 \pm 4.32 ; p<$ 0.01 ) (Figure 2), mean negative scale $(29.53 \pm 3.64$ vs. $24.77 \pm 3.23 ; p<0.01)$ (Figure 3), and mean general psychopathology scale $(57.75 \pm 6.24$ vs. 48.15 $\pm 5.79 ; p<0.01$ ) (Figure 4). The items that took one week to start improving were the mean total score $(117.38 \pm 11.74$ vs. $105.66 \pm 11.75 ; p<0.01)$, the mean positive scale $(30.09 \pm 4.18$ vs. $23.81 \pm$ $4.50 ; p<0.01)$ and the mean general psychopathology scale $(57.75 \pm 6.24$ vs. $53.69 \pm 6.21 ; p<0.05)$, and 2 weeks was required for the mean negative scale $(29.53 \pm 3.64$ vs. $28.16 \pm 3.65 ; p<0.05)$ (Figure 1 to 4 ).

\section{Discussion and Conclusion}

In this study, it is meaningful that there was significant improvement in all four items of the total score, positive scale, negative scale and general psychopathology scale after 4 weeks. However, it is especially noteworthy that the efficacy of BPZ was rapid, taking only one week for the total score and the positive and general psychopathology scales, and 2 weeks for the negative scale.

Previous studies have shown similar results. One study assessed BPZ with the PANSS in the same way as in the present study, but with different doses (2-4 mg/day) and duration (6 weeks). The results showed a rapid improvement in the positive and negative scales, as in the present study. This study differs from ours in that it concluded that there was no improvement in cognitive function [3].

Some studies suggest that BPZ may be effective in improving cognitive function. One study examined the effects and side effects of BPZ on agitation in Alzheimer's disease. In this study, BPZ was prescribed in doses of 0.5 to $2.0 \mathrm{mg}$ and the results were assessed using the Cohen-Mansfield Agitation Inventory (CMAI) CohenMansfield Agitation Inventory (CMAI) [4]. This study shows that the prescription of BPZ $2.0 \mathrm{mg}$ is effective in the agitation of Alzheimer's disease. Although not approved in Japan, BPZ has been shown to be effective in the treatment of treatment-resistant major depression [5] and bipolar depression [6], which do not respond to other antidepressants.

There are also reports that BPZ is effective for attention-deficit/ hyperactivity disorder and posttraumatic stress disorder associated with domestic violence [7-8], but these remain at the level of case studies and require further research. 
We then consider the efficacy and side effects of BPZ, which have been reported in a wide range of previous studies, some comparing BPZ with other antipsychotics. The efficacy of BPZ in the acute phase of schizophrenia is comparable to other second-generation antipsychotics such as quetiapine, aripiprazole and ziprasidone [9].

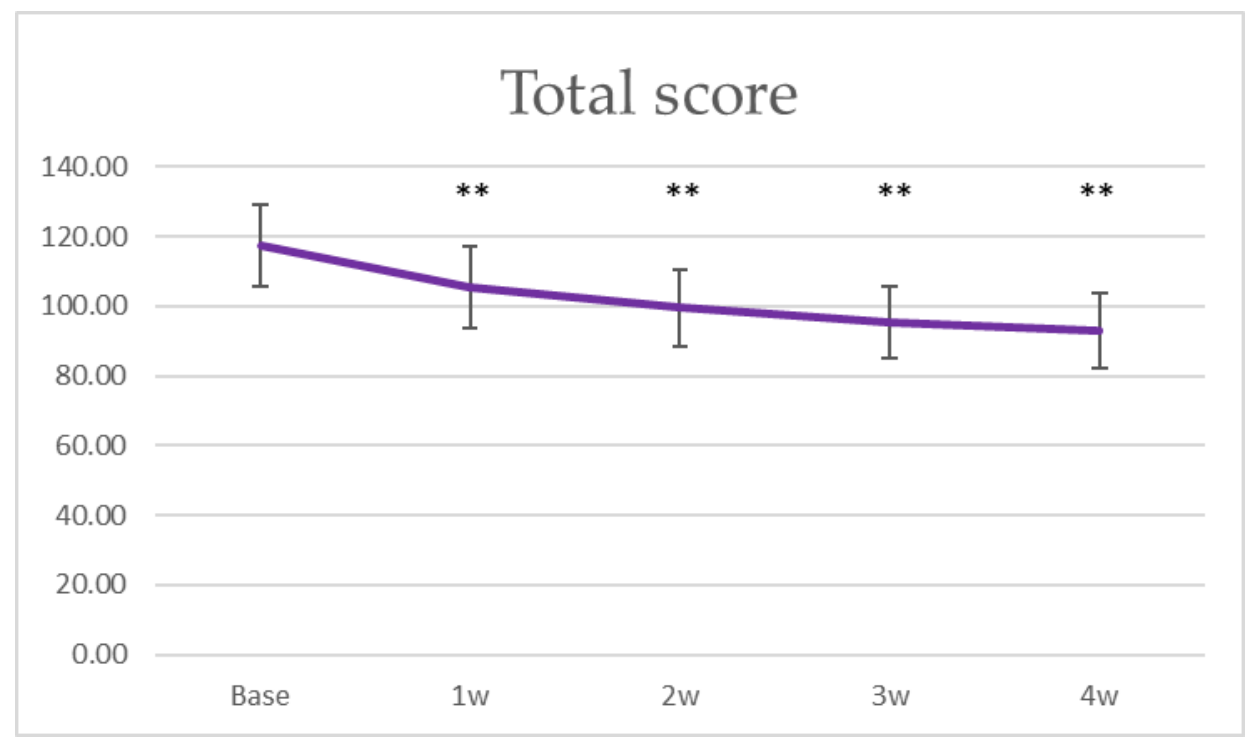

Figure 1. The graph shows the changes in the total score in PANSS. * Significantly improved over base $(p<0.05)$. ${ }^{* *}$ Significantly improved over base $(p<0.01)$.

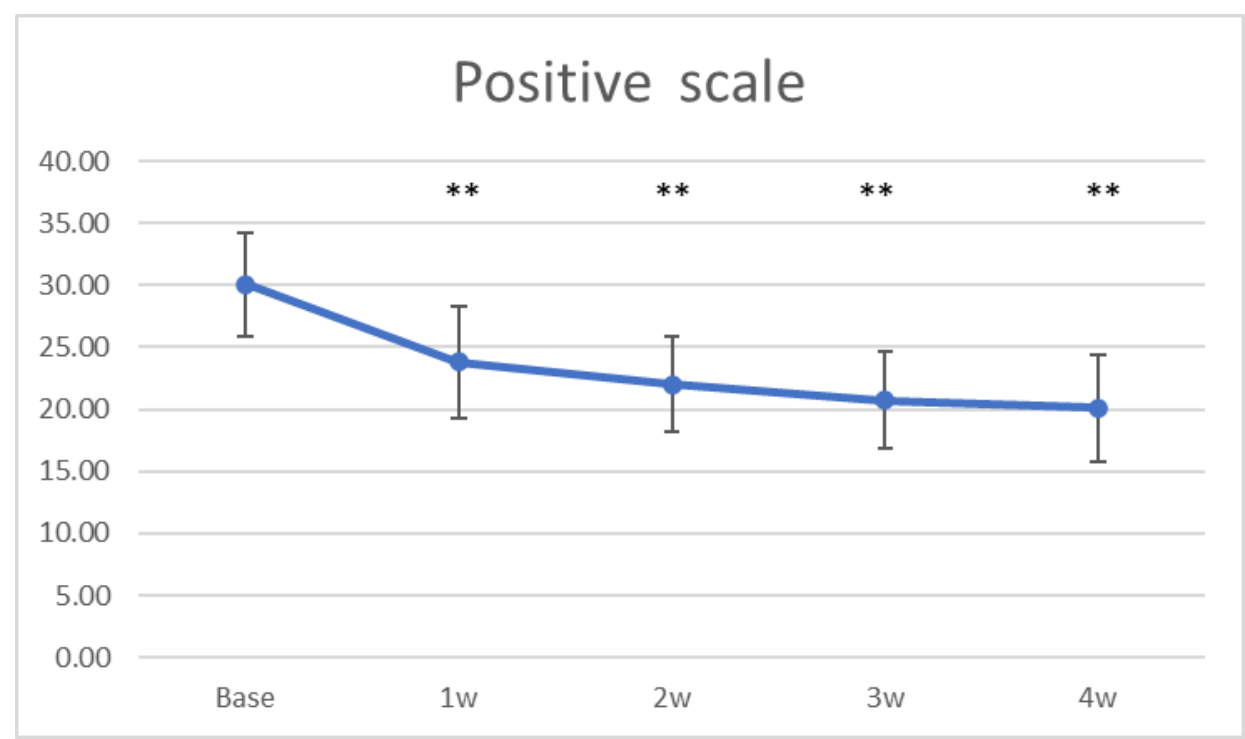

Figure 2. The graph shows the changes in the positive scale in PANSS. * Significantly improved over base $(p<0.05)$. ${ }^{*}$ Significantly improved over base $(p<0.01)$. 


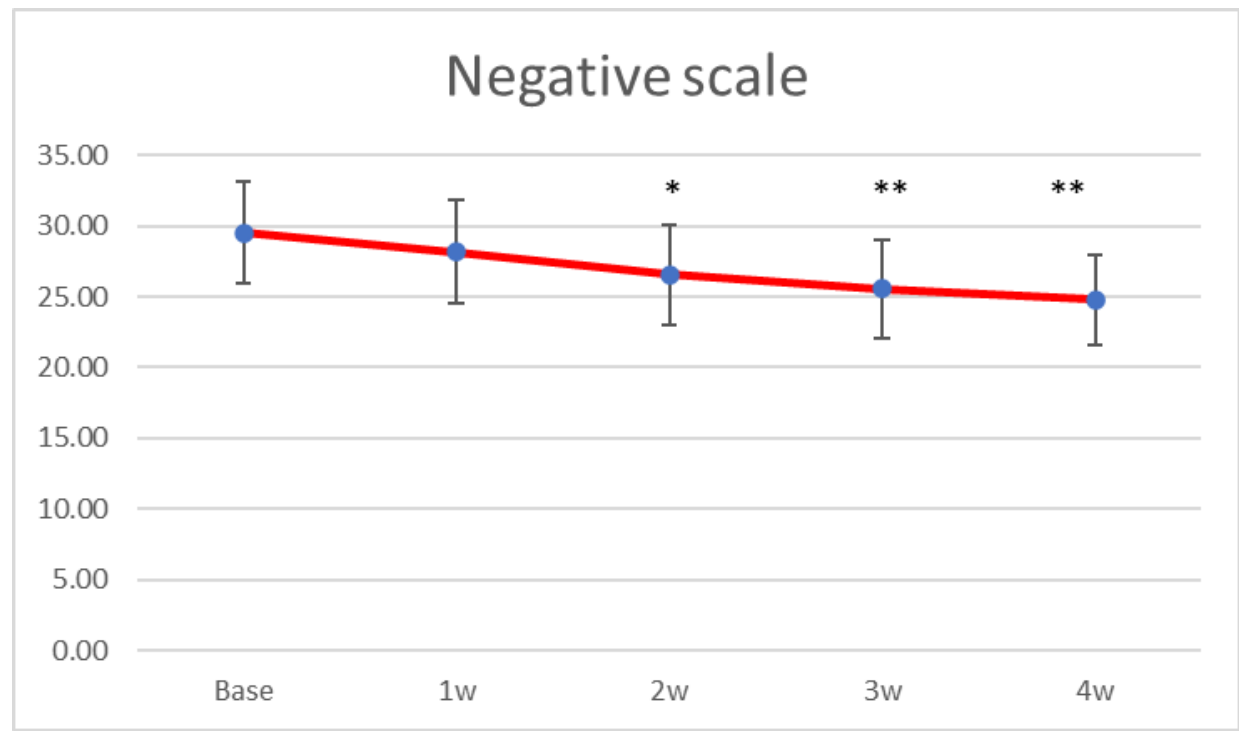

Figure 3. The graph shows the changes in the negative scale in PANSS. * Significantly improved over base $(p<0.05)$. ${ }^{* *}$ Significantly improved over base $(p<0.01)$.

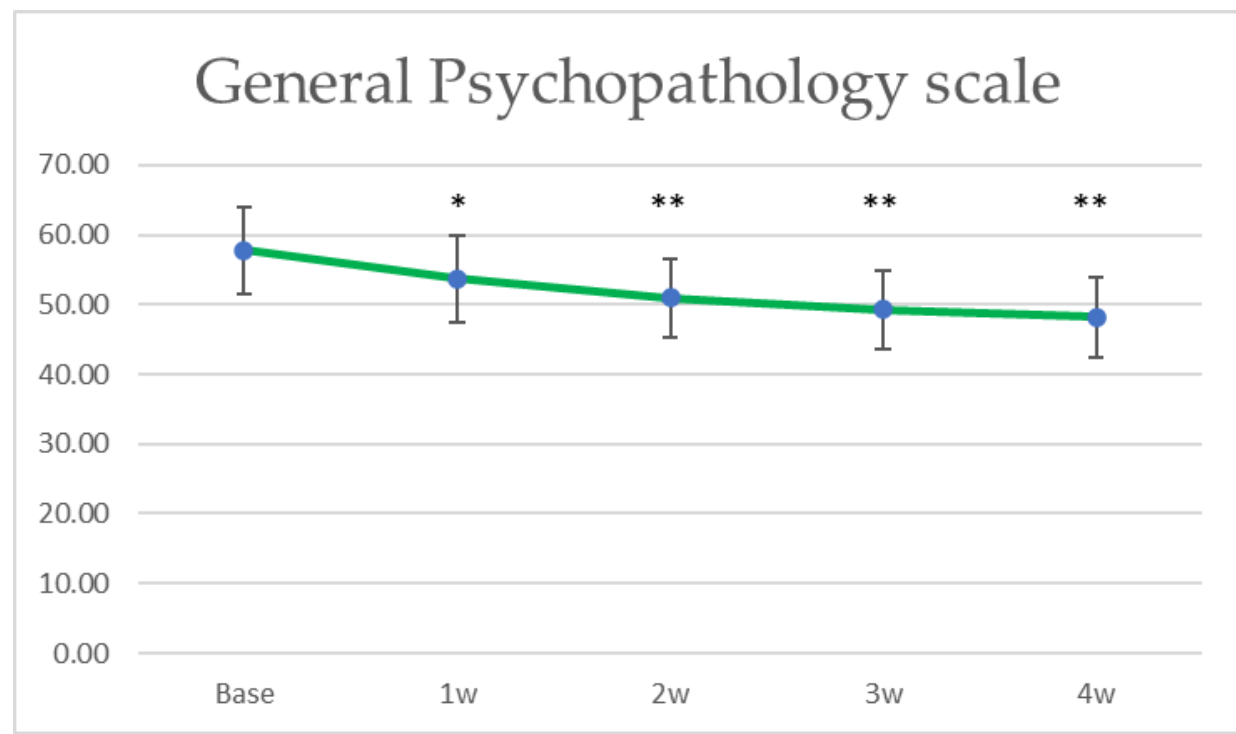

Figure 4. The graph shows the changes in the general psychopathology scale in PANSS. * Significantly improved over base $(p<0.05)$. * Significantly improved over base $(p$ $<0.01)$

It has also been reported to improve symptoms of schizophrenia as well as prevent relapses [10] and improve depressive symptoms [11]. In terms of side effects, it has been reported to have fewer metabolic and cardiovascular side effects [9] and to reduce the risk of extrapyramidal symptoms, hyperprolactinaemia, weight gain, psychosis, insomnia, nausea/vomiting or restlessness [10]. 
However, there are both high [9, 11] and low [9] reports of risk for akathisia, and caution should be exercised when using BPZ in clinical practice.

The continuation rate of $\mathrm{BPZ}$ in this study was $32 / 41=78.0 \%$. How about the continuation rate of BPZ in the longer term? Two previous studies have answered this question: one showed a $\mathrm{BPZ}$ retention rate of $68 / 120=57.7 \%$ after 16 weeks [12], and the other showed a retention rate of $38.6 \%$ after 52 weeks [13]. BPZ, like other antipsychotics, requires medication supervision by psychiatrists and comedical staff.

The limitation of this study is that it was a non-blind study. Future studies should include analyses of patient characteristics such as age and gender, $\mathrm{BPZ}$ doses, and more sub-items of the positive and negative scales and the general psychopathology scale.

\section{References}

[1] Otsuka Pharmaceutical Company. Otsuka and Lundbeck's Rxulti ${ }^{\circledR}$ (brexpiprazole) receives positive opinion in EU from CHMP for the treatment of schizophrenia in adults. Available in: https://www.otsuka.co.jp /en/company/newsreleases/2018/201806 04_1.html

[2] Hope J, Castle D, Keks NA. Brexpiprazole: a new leaf on the partial dopamine agonist branch. Australas
Psychiatry. 2018 Feb;26(1):92-94. doi: $10.1177 / 1039856217732473$.

[3] Leucht S, Crippa A, Siafis S, Patel MX, Orsini N, Davis JM. Dose-Response Meta-Analysis of Antipsychotic Drugs for Acute Schizophrenia. Am J Psychiatry. 2020 Apr 1;177(4):342-353. doi: 10.1176/appi.ajp.2019.19010034.

[4] Cohen - Mansfield J. Conceptualization of agitation: results based on the Cohen-Mansfield Agitation Inventory and the Agitation Behavior Mapping Instrument. Int Psychogeriatr. 1996;8 Suppl 3:309-15; discussion 351-4. doi: $10.1017 / \mathrm{s} 1041610297003530$.

[5] Fornaro M, Fusco A, Anastasia A, Cattaneo CI, De Berardis D. Brexpiprazole for treatment-resistant major depressive disorder. Expert Opin Pharmacother. 2019 Nov;20(16):1925$1933 . \quad$ doi: 10.1080/ 14656566.2019.1654457.

[6] Brown ES, Khaleghi N, Van Enkevort E, Ivleva E, Nakamura A, Holmes T, Mason BL, Escalante C. A pilot study of brexpiprazole for bipolar depression. J Affect Disord. 2019 Apr 15;249:315-318. doi: 10.1016/j.jad.2019.02. 056.

[7] Howland RH. Brexpiprazole: another multipurpose antipsychotic drug? Journal of Psychosocial Nursing and Mental Health Services. 2015 Apr;53(4):23-5. doi: 10.3928/0279369520150323-01.

[8] O'Connor M. Adjunctive therapy with brexpiprazole improves treatment 
resistant complex post traumatic stress disorder in domestic family violence victims. Australasian Psychiatry. 2020 Jun;28(3):264-266. doi: 10.1177/ 1039856219889303.

[9] Corponi F, Fabbri C, Bitter I, Montgomery S, Vieta E, Kasper S, Pallanti S, Serretti A. Novel antipsychotics specificity profile: A clinically oriented review of lurasidone, brexpiprazole, cariprazine and lumateperone. Eur Neuropsychopharmacol. 2019 Sep;29(9):971-985. doi: 10.1016/j.euroneuro.2019.06.008.

[10] Watanabe Y, Yamada S, Otsubo T, Kikuchi T. Brexpiprazole for the Treatment of Schizophrenia in Adults: An Overview of Its Clinical Efficacy and Safety and a Psychiatrist's Perspective. Drug Des Devel Ther. 2020 Dec 18;14:5559-5574. doi: 10.2147/ DDDT.S240859.

[11] Antoun Reyad A, Girgis E, Mishriky R. Efficacy and safety of brexpiprazole in acute management of psychiatric disorders: a meta-analysis of randomized controlled trials. Int Clin Psychopharmacol. 2020 May;35(3):119128. doi: 10.1097/YIC.0000000000000308.

[12] Yoshimura $\mathrm{Y}$, Shimizu $\mathrm{H}$, Yamashita R, Washida K, Takeda T, Aoki S. Association between previous high-dose antipsychotic therapy and brexpiprazole discontinuation after the initiation of brexpiprazole in patients with schizophrenia or schizoaffective disorder. Int Clin Psychopharmacol. 2020 Mar;35(2):98-104. doi: 10.1097/YIC.0000000000000296.

[13] Inoue $Y$, Suzuki $H$, Hibino $H$, Takaya A, Mikami K, Yamamoto K, Matsumoto $H$. Continuation rate for asenapine and brexpiprazole treatment in patients with schizophrenia. Brain Behav. 2021 May;11(5):e02109. doi: 10.1002/brb3.2109.

Conflict of interest: The authors declare no conflicts of interest associated with this manuscript. The patients have provided permission to publish these features including his examination data, and the identity of the patients have been protected.

Acknowledgements: We thank Drs. Kobayashi Seiju and Shirasaka Tomonobu for helpful discussions and comments on the manuscript.

Funding: None.

How to cite this article: Tetsuro I, Tomonori M. Investigation of the effect of brexpiprazole on acute or relapse schizophrenia using the positive and negative syndrome scale: an experimental report. Brazilian Journal of Case Reports. 2022 Jan-Mar;02(1):83-89. 